\title{
Effects of Attention and Emotion on Repetition \\ Priming and Their Modulation by Cholinergic \\ Enhancement
}

Paul Bentley, Patrik Vuilleumier, Christiane M. Thiel, Jon Driver and Raymond J. Dolan

$J N$ 90:1171-1181, 2003. First published Mar 20, 2003; doi:10.1152/jn.00776.2002

You might find this additional information useful...

This article cites 63 articles, 20 of which you can access free at:

http://jn.physiology.org/cgi/content/full/90/2/1171\#BIBL

This article has been cited by 3 other HighWire hosted articles:

Selective Attention Modulates Neural Substrates of Repetition Priming and "Implicit"

Visual Memory: Suppressions and Enhancements Revealed by fMRI

P. Vuilleumier, S. Schwartz, S. Duhoux, R. J. Dolan and J. Driver

J. Cogn. Neurosci., August 1, 2005; 17 (8): 1245-1260.

[Abstract] [Full Text] [PDF]

BOLD Repetition Decreases in Object-Responsive Ventral Visual Areas Depend on Spatial Attention

E. Eger, R.N.A. Henson, J. Driver and R. J. Dolan

J Neurophysiol, August 1, 2004; 92 (2): 1241-1247.

[Abstract] [Full Text] [PDF]

Repetition suppression of faces is modulated by emotion

A. Ishai, L. Pessoa, P. C. Bikle and L. G. Ungerleider

$P N A S$, June 29, 2004; 101 (26): 9827-9832.

[Abstract] [Full Text] [PDF]

Updated information and services including high-resolution figures, can be found at:

http://jn.physiology.org/cgi/content/full/90/2/1171

Additional material and information about Journal of Neurophysiology can be found at: http://www.the-aps.org/publications/jn

This information is current as of March 29, 2006.

Journal of Neurophysiology publishes original articles on the function of the nervous system. It is published 12 times a year (monthly) by the American Physiological Society, 9650 Rockville Pike, Bethesda MD 20814-3991. Copyright (C) 2005 by the American Physiological Society. ISSN: 0022-3077, ESSN: 1522-1598. Visit our website at http://www.the-aps.org/. 


\title{
Effects of Attention and Emotion on Repetition Priming and Their
}

\section{Modulation by Cholinergic Enhancement}

\author{
Paul Bentley, ${ }^{1}$ Patrik Vuilleumier, ${ }^{2}$ Christiane M. Thiel, ${ }^{1,3}$ Jon Driver, ${ }^{2}$ and Raymond J. Dolan ${ }^{1}$ \\ ${ }^{1}$ Wellcome Department of Imaging Neuroscience, London WC1N 3BG; ${ }^{2}$ Institute of Cognitive Neuroscience, London WC1N 3AR, \\ United Kingdom; and ${ }^{3}$ Institut für Medizin (IME), Forschungszentrum Jülich, 52425 Jülich, Germany
}

Submitted 9 September 2002; accepted in final form 15 February 2003

Bentley, Paul, Patrik Vuilleumier, Christiane M. Thiel, Jon Driver, and Raymond J. Dolan. Effects of attention and emotion on repetition priming and their modulation by cholinergic enhancement. J Neurophysiol 90: 1171-1181, 2003. First published March 20, 2003; 10.1152/jn.00776.2002. We examined whether behavioral and neural effects of repeating faces are modulated by independent factors of selective attention, emotion, and cholinergic enhancement, during functional MRI. Face repetition occurred either between task-relevant (spatially attended) or task-irrelevant (unattended) stimuli; faces could be fearful or neutral; subjects received either placebo or physostigmine. Under placebo, a reaction time advantage occurred with repetition (i.e., priming) that did not differ between levels of attention, but was attenuated with emotion. Inferior temporo-occipital cortex demonstrated repetition decreases to both attended and unattended faces, and showed either equivalent or greater repetition decreases with emotional compared with neutral faces. By contrast, repetition decreases were attenuated for emotional relative to neutral faces in lateral orbitofrontal cortex. These results distinguish automatic repetition effects in sensory cortical regions from repetition effects modulated by emotion in orbitofrontal cortex, which parallel behavioral effects. Under physostigmine, unlike placebo, behavioral repetition effects were seen selectively for attended faces only, whereas emotional faces no longer impaired priming. Physostigmine enhanced repetition decreases in inferior occipital cortex selectively for attended faces, and reversed the emotional interaction with repetition in lateral orbitofrontal cortex. Thus we show that cholinergic enhancement both augments a neural signature of priming and modulates the effects of attention and emotion on behavioral and neural consequences of repetition.

\section{N T R O D U C T I O N}

Stimulus repetition is associated with decreases in cortical activity (Buckner et al. 1998), which may reflect more efficient stimulus processing and underlie perceptual priming (Schacter and Buckner 1998). In sensory-perceptual areas, where this effect occurs most robustly (Badgaiyan 2000), the degree to which the underlying neural mechanisms operate automatically (Desimone 1996; Wiggs and Martin 1998), versus being influenced by top-down factors (Henson et al. 2002), remains unclear. Similar repetition decreases for both target and foil faces have been observed in extrastriate visual cortex during a working memory task (Jiang et al. 2000), and in superior temporal gyrus to written words after both divided and full attention study phases using spoken words (Badgaiyan et al.

\footnotetext{
Address for reprint requests: P. Bentley, Wellcome Department of Imaging Neuroscience, 12 Queen Square, London WC1N 3BG, UK (E-mail: p.bentley@fil.ion.ucl.ac.uk).
}

2001). Nevertheless, differences in face-repetition effects in early visual areas have been observed depending on task (Henson et al. 2002; Reber et al. 1998), in keeping with electrophysiological data (Dale et al. 2000; Puce et al. 1999), suggesting that sensory repetition effects may be influenced by reentrant signals from regions involved in higher levels of processing. If repetition effects in sensory cortex are entirely dependent on task-related processing (e.g., in prefrontal cortex), then it might be expected that stimuli that are completely irrelevant to the task (at both study and test) would not engender repetition effects. Our first aim in the current experiment was to determine whether repetition effects in extrastriate visual cortex, as measured by functional MRI (fMRI), would be observed for faces both when selectively attended and ignored as distractors.

A further factor that may influence priming, independently of attention, is the emotional value of a stimulus. Emotional stimuli can enhance activity within extrastriate visual areas separately from an effect of attention (Vuilleumier et al. 2001), which appears to be related to association learning in the context of fear conditioning (Morris et al. 2001). Because priming may represent a similar form of perceptually based implicit memory (Schacter and Buckner 1998), it might also be expected to be enhanced by emotion. Thus behavioral studies suggest a priming benefit for emotional stimuli in healthy adults (LaBar and Phelps 2002), as well as depressed and social phobic patients (Lundh and Ost 1997; Watkins et al. 2000). On the other hand, unpleasant (vs. neutral) faces have been found in one fMRI study to result in less of a repetition decrease in temporo-occipital cortex, which was interpreted in terms of reduced adaptation to negative valence stimuli (Rotshtein et al. 2001). An alternative explanation, however, was that the critical faces in this particular study were not just unpleasant but also bizarre (i.e., mouth and eyes were inverted), which could have influenced repetition effects through indirect attentional factors. In the present experiment we examined the effect of face-emotion on repetition effects, orthogonal to effects of attention, by using an event-related design that repeated faces only once so as to minimize habituation (Brown and Xiang 1998).

In the second part of this study, we examined whether cholinergic enhancement would modulate behavioral and fMRI

\footnotetext{
The costs of publication of this article were defrayed in part by the payment of page charges. The article must therefore be hereby marked "advertisement" in accordance with 18 U.S.C. Section 1734 solely to indicate this fact.
} 
repetition effects in a separate group of subjects given the anticholinesterase physostigmine. Behavioral and hemodynamic measures of priming have both previously been found to be impaired by cholinergic blockade with scopolamine (Thiel et al. 2001, 2002a), in line with known effects of acetylcholine on cortical plasticity and learning (e.g., Rasmusson 2000). By contrast, cholinergic enhancement may improve an fMRI measure of perceptual processing in extrastriate cortex, selectively for stimuli that must be remembered (Furey et al. 2000a), while increasing the behavioral effect of word-priming in Alzheimer's disease (Riekkinen and Riekkinen 1999). On this basis, plus the proposal that cholinergic modulation may favor processing selectively of attended stimuli (Sarter et al. 2001), we hypothesized that physostigmine would result in greater neural and behavioral repetition effects specifically for attended (taskrelevant) stimuli. Furthermore, because acetylcholine enhances cortical plasticity specifically for emotional stimuli, as in fearconditioning (Ji et al. 2001; Thiel et al. 2002b; Weinberger et al. 1998), we predicted that priming effects to fearful, relative to neutral, faces would be greater under physostigmine.

\section{METHODS}

\section{Subjects}

Thirty healthy right-handed volunteers with no history of medical or psychiatric disease gave written informed consent. They were divided into 2 groups of 15 (placebo: 7 female, 8 male; mean age $=$ $26.8 \pm 2.3$; physostigmine: 8 female, 7 male; mean age $=23.5 \pm$ 2.0). No subject was taking active medication. Although 2 subjects were mild smokers, they were in different groups, and refrained from smoking on the test day. A between-subjects design was chosen for the pharmacological manipulation, to exclude any between-session repetition effects (e.g., van Turrennout et al. 2000), and to minimize any long-term habituation to emotional stimuli (e.g., Thiel et al. 1999).

\section{Cognitive task}

Subjects performed a matching task (after Vuilleumier et al. 2001) for 2 black and white photographs situated in either the north-south or east-west coordinates of a cross-format display that consisted of 4 concurrent photographs $\left(3^{\circ} \times 5^{\circ}\right.$ visual angle each), arranged into a cross around a central fixation point (Fig. 1). At the start of each block, subjects were cued (for $2 \mathrm{~s}$ ) to attend selectively to either the 2 vertically arranged or 2 horizontally arranged positions, whereas the alternative 2 locations were to be ignored throughout the block. Thus attention was directed on the basis of spatial location, rather than on the basis of stimulus type per se (face vs. house). In total there were 4 blocks of 40 trials each. Each trial consisted of a central fixation cross (1 s) followed by the 4-picture display for $250 \mathrm{~ms}$. Subjects were required to indicate, as accurately and rapidly as possible, whether the 2 stimuli at task-relevant locations were the same or different, by either of 2 possible key presses with the right hand. Reaction time (RT) and accuracy were recorded. The median intertrial interval was $2.5 \mathrm{~s}$ (range: $1.5-14.4 \mathrm{~s}$ ).

Within each trial, either the 2 attended or the 2 unattended locations were occupied by 2 nonfamous faces (taken from The Karolinska Directed Emotional Faces set; Lundqvist et al. 1998), in an intermingled and unpredictable sequence. The remaining 2 locations were occupied by 2 house pictures; hence a trial could be classified as faces-attended (A) or faces-unattended (U), depending on the faces' position with respect to the currently attended locations (i.e., attention was spatially based, and we use the terminology of "faces-attended," for instance, only as a shorthand for "faces-appearing-at-the-attendedlocations"). Furthermore, both faces had either a fearful emotion (E) or neutral $(\mathrm{N})$ expression, independent of whether they were at taskrelevant locations. This resulted in 4 conditions (AE, AN, UE, and UN faces); these conditions plus the pair identities (i.e., same/different, which was independent between the attended and unattended pair in each trial) were randomly counterbalanced throughout each block. The order of task-relevant locations (i.e., either vertical or horizontal) between blocks was randomly picked from one of 4 alternatives (HVHV, VHVH, HVVH, VHHV), and counterbalanced across subjects within each group.

Stimulus repetition occurred for only particular face stimuli. Every

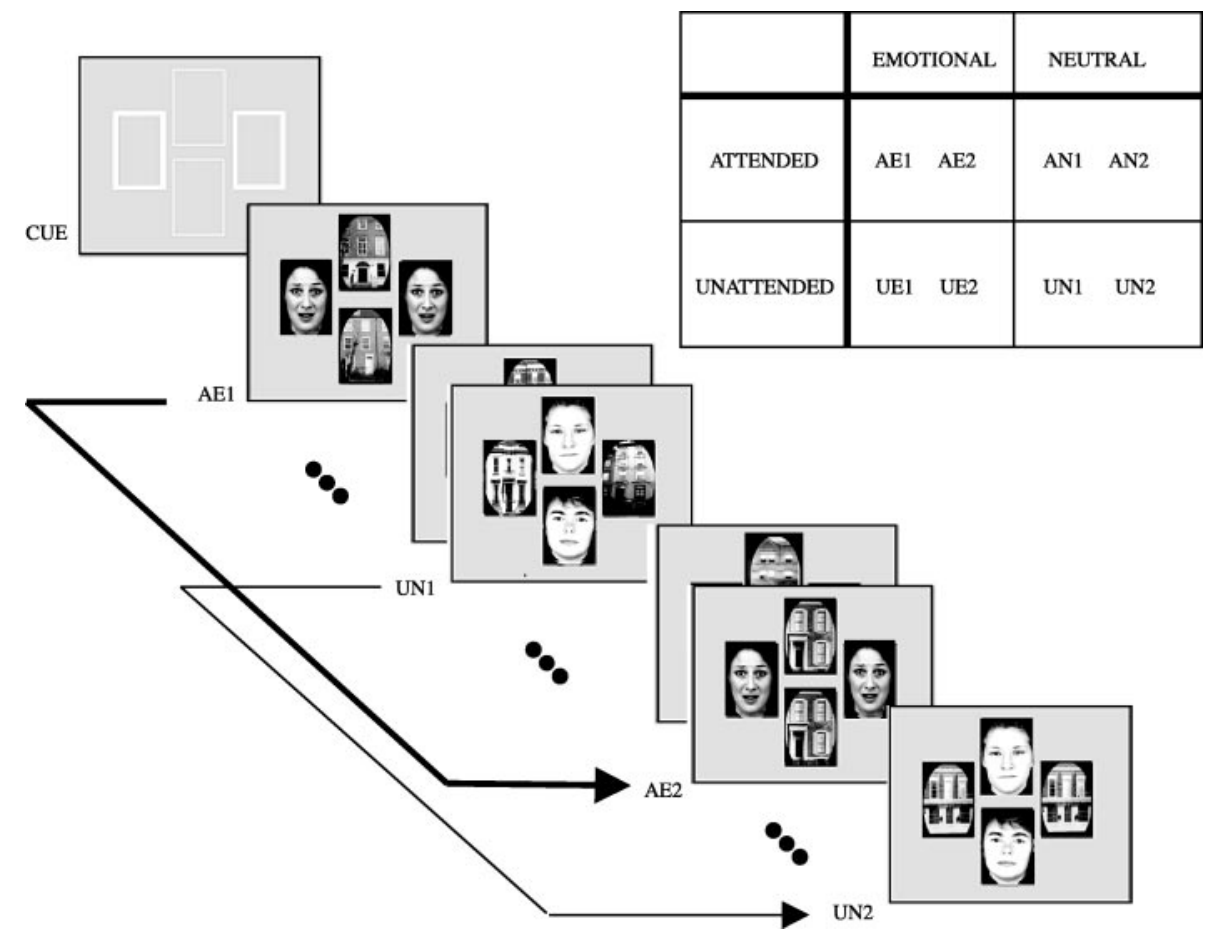

FIG. 1. Stimulus format. Each block started with cue stimulus that indicated whether subjects must attend to horizontal or vertical locations in cross-array for performance of subsequent matching task (in this example, this would be horizontal locations). Each trial consisted of a pair of faces that may be either in task-relevant (A) or taskirrelevant (U) locations; together with a pair of houses occupying alternate 2 locations. Face pairs were repeated with lag of 2 to 5 intervening trials; houses were never repeated. Face pairs could be either fearful and thus emotional $(\mathrm{E})$ or neutral $(\mathrm{N})$. Experiment thus had a $2 \times 2 \times 2$ factorial design, with factors of face-repetition, attention (toward or away from faces), and emotion. 
first presentation of a pair of faces (suffixed 1 henceforth) was followed by only one other repetition of the same pair of faces (suffixed 2) that occurred after an interval of 2 to 5 intervening trials (with approximately equal distribution across each block). Thus 8 conditions existed: AE1, AE2, AN1, AN2, UE1, UE2, UN1, and UN2 (where AE1, for example, would represent the first presentation of a particular pair of fearful faces appearing at task-relevant locations, and AE2 would represent their subsequent repetition). There were 20 trials of each condition per subject. Because of the small number of intervening stimuli between first and second repetitions, the influence of any time confound is minimal $(<5 \%$ trials fell outside of the time window in which stimulus order was fully counterbalanced with respect to time).

The cross-format spatial array and brief exposure time has previously been shown to be effective at engaging covert attention to the relevant pair of locations without saccades (Vuilleumier et al. 2001; Wojciulik et al. 1998), and without awareness of identity, gender, or expression of faces when these appear in task-irrelevant locations only (Vuilleumier et al. 2001). Nonetheless, to exclude possible betweengroup differences in saccade frequency or compliance with the requirement for central fixation, we monitored eye movements throughout the task using an infrared eye tracker (ASL Model 540, Applied Science Group, Bedford, MA; refresh rate $=60 \mathrm{~Hz}$ ). For technical reasons, eye-position data were lost for 6 subjects ( 2 placebo, 4 from drug group).

\section{Drug treatment}

A double-blind placebo-controlled drug administration technique was used. Each subject received an intravenous cannula into the left cubital fossa and an infusion of either physostigmine or saline. Dosage and rate of physostigmine infused was identical to that used in a recent study (Furey et al. 2000b), providing stable levels of plasma drug concentration and butyrylcholinesterase inhibition, as well as significant and stable effects on cognitive performance for $40 \mathrm{~min}$, after a 40-min loading period. Furthermore, the same protocol was also found to result in changes in both regional cerebral blood flow (rCBF) and blood oxygenation level-dependent (BOLD) activity, during visual working memory tasks (Furey et al. 1997, 2000a).

Subjects in the drug group first received $0.2 \mathrm{mg}$ intravenous glycopyrrolate, a peripheral muscarinic receptor antagonist, to reduce peripheral side effects. The placebo group were injected with an equivalent volume of saline. Both groups then received an intravenous infusion. In the case of the drug group this consisted of physostigmine at a rate of $1.93 \mathrm{mg} / \mathrm{h}$ for $10 \mathrm{~min}$, followed by a constant rate of 0.816 $\mathrm{mg} / \mathrm{h}$ for $40 \mathrm{~min}$, before scanning. The same rate was then continued until the end of study so that each subject received no more than 1.3 $\mathrm{mg}$ physostigmine in total. The placebo group received an equivalent volume of saline over the same time course. Both groups of subjects had their blood pressure checked before and at 40 min into infusion; pulse-oximetry was performed continuously throughout the experiment. Subjects were given a questionnaire at both time points that allowed a ranked measurement ( $0-6$ scale) of 7 recognized adverse reactions to physostigmine and glycopyrrolate, as well as a list of visual analog scales for estimating subjective feelings (Bond and Lader 1974).

\section{Imaging and image processing}

MRI data were acquired from a $2 \mathrm{~T}$ VISION system (Siemens, Erlangen, Germany) equipped with a head coil. Functional images were acquired with a gradient echo-planar T2* sequence using BOLD contrast. The acquired image consisted of $32 \times 3$-mm-thick axial slices that covered the entire brain. Volumes were acquired in a single continuous session with an effective repetition time of $3.26 \mathrm{~s}$. The first 8 volumes were discarded, to allow for T1 equilibration effects. Images were realigned, time-corrected, normalized to a standard echo- planar image template, and smoothed with a Gaussian kernel of 8-mm full-width half-maximum.

\section{Statistical analysis of images}

Data were analyzed with a general linear model for event-related designs (SPM99; Wellcome Dept. of Cognitive Neurology, London, UK; Friston et al. 1995) using a random-effects analysis. Data were globally scaled and high-pass filtered at 1/120 Hz. Eight event types were defined for each subject (see above). Individual events were modeled by a canonical synthetic hemodynamic response function and its temporal derivative (Friston et al. 1998). The 6 head movement parameters were included within the model as confounding covariates.

Linear contrasts of parameter estimates were made for each subject and used to generate statistical parametric maps (SPMs) of the $t$ statistic. To test for regions showing face-repetition effects common to different conditions (i.e., to both attended and unattended faces; or to both emotional and neutral faces), contrasts of first versus second presentation under each condition were performed for each subject and entered into a repeated-measures analysis of variance (ANOVA) corrected for nonsphericity (Glaser et al. 2002). A conjunction analysis was then performed over contrasts from both conditions (Price and Friston 1997). To test for regions showing a different magnitude of repetition decrease between conditions, contrasts representing the repetition $\times$ condition interaction for each subject were entered into a one-sample $t$-test; only regions showing a significant repetition decrease $(P<0.001$, uncorrected $)$ in at least one of the tested conditions are reported. Regions found to show repetition $\times$ condition interactions that also showed a significant 3-way interaction (repetition $\times$ attention $\times$ emotion; thresholded at $P<0.001$, uncorrected) are noted.

Repetition $\times$ drug interactions were analyzed separately for attended and unattended faces by comparing between-subject repetition effects with $t$-tests. Repetition $\times$ condition $\times$ drug interactions were similarly assessed by comparing the repetition $\times$ condition interactions, for each subject, between groups, but only the volume of voxels showing a repetition main effect or repetition $\times$ condition interaction in the placebo group was searched (masks thresholded at $P<0.001$, uncorrected). Any effects of physostigmine on attention and emotion, independent of repetition, are considered elsewhere (Bentley et al. 2003). We emphasize that the drug effects reported here are condition specific, as mean session effects are modeled separately. All regions that showed significant repetition $\times$ drug, or repetition $\times$ condition $\times$ drug, interactions were found to show insignificant between-group session effects $(P>0.05$, uncorrected). Furthermore, the global session mean activity did not differ between groups $(P>0.05)$, suggesting that physostigmine did not engender significant general vascular effects.

We report areas that achieve significance either after correction for whole brain (or effective search volume in the case of repetition $\times$ condition $\times$ drug interactions; see above), or regions of interest (ROI) where indicated (Worsley et al. 1996). Two ROIs of approximately 20 $\mathrm{cm}^{3}$ each were defined (Rorden and Brett 2001) in right and left inferior temporo-occipital cortices so as to encompass coordinates previously found to show repetition decreases in event-related fMRI designs for repetition of unfamiliar faces (Henson et al. 2002; Jiang et al. 2001). Regions surviving a threshold of $P<0.001$, uncorrected, are also reported descriptively. Any activations smaller than 5 contiguous voxels were discounted.

\section{RES U L T S}

\section{Behavioral}

Behavioral effects of priming were determined by calculating median RT and mean accuracy differences between the first and second presentations for each face pair, separately for each 
of the 4 conditions in each participant. In the placebo group, a significant speeding of responses when attended neutral (AN) faces were repeated [mean RT difference between first and second presentation $=30.3 \pm 17.5 \mathrm{~ms}, t(14)=3.5, P<$ 0.005], plus a trend for more accurate performance with repeated stimuli [mean accuracy difference $=5.3 \pm 5.8 \%$; $t(14)=1.8, P<0.1]$ was evident, consistent with previous studies of repetition priming (e.g., Henson et al. 2002).

To ascertain any effects of condition (attention and emotion) and group (cholinergic enhancement vs. placebo), we entered RT and accuracy differences (between first and second presentation) for each subject, for each condition, into a repeatedmeasures, mixed ANOVA. Although there was no main effect of group on the RT priming effect, there were significant group $\times$ attention $[F(1,28)=4.4 ; P<0.05]$ and group $\times$ emotion interactions $[F(1,28)=4.3 ; P<0.05 ;$ Fig. $2 A]$. These represented a significant reduction of the RT priming effect for emotional versus neutral trials under placebo $[F(1,14)=11.7$; $P<0.005]$, but not under physostigmine $[F(1,14)=0.7 ; \mathrm{ns}]$, whereas the RT priming effect was significantly greater for attended versus unattended faces under physostigmine $[F(1,14)=12.8 ; P<0.005]$, but not under placebo $[F(1,14)=$ 0.3 ; ns]. A separate ANOVA comparing absolute RTs between groups over condition (attention $\times$ emotion $\times$ stimulus order)

A

RT

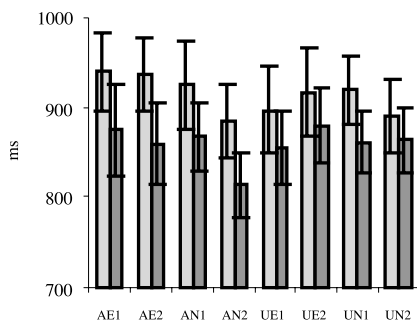

口Placebo $\square$ Physostigmine

B Accuracy

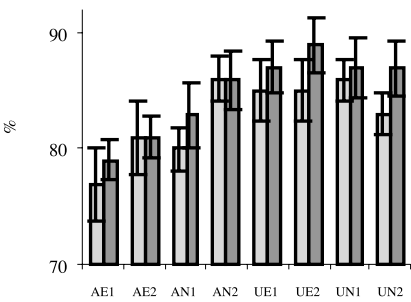

口Placebo $\square$ Physostigmine
RT : 1st - 2nd

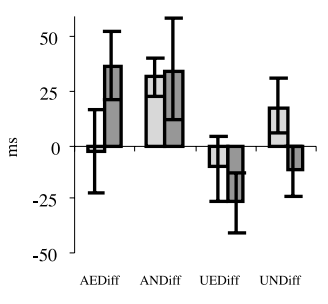

口Placebo QPhysostigmine

Accuracy: 2nd-1st

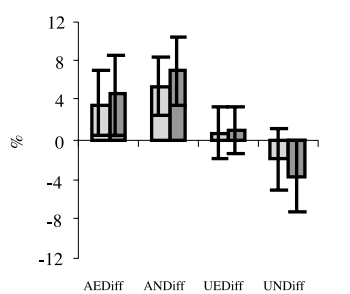

DPlacebo DPhysostigmine

FIG. 2. A: median RT for each of 8 conditions (AE, Attended Emotional faces; AN, Attended Neutral; UE, Unattended Emotional; UN, Unattended Neutral; -1 : 1st presentation; -2 : 2nd presentation), separately for placebo and physostigmine groups. Second graph shows itemwise differences in RTs to novel minus primed stimuli for each of 4 main conditions (hence positive values represent repetition advantage). $B$ : mean accuracy for same 8 conditions. Second graph shows itemwise differences of accuracy to primed minus novel stimuli for each of 4 main conditions (hence positive values represent repetition advantage). SE bars are shown. Note that statistical inferences concerning priming are based on subject-specific differences, and that inferences on behavioral effects are based on item-specific repetition (not conveyed by error bars on group means).

did not reveal any between-group differences, although there was a trend to faster overall RTs in the physostigmine group $[t(28)=1.4, P<0.1,1$-tailed based on Furey et al. 1997].

There was no group $\times$ condition interaction for priming in accuracy measures, but a trend for greater priming with attended repeated faces versus unattended repeated faces $[F(1,14)=3.0 ; P<0.1$; Fig. $2 B]$. Overall accuracy (mean score $=85 \pm 3.2 \%$, and $83 \pm 3.2 \%$, under drug and placebo, respectively) was comparable to that of a previous study using the same task in which limited awareness of task-irrelevant compared with task-relevant faces was demonstrated (Vuilleumier et al. 2001). There were no behavioral effects of the specific attended location (i.e., horizontal or vertical pair), nor any interactions of location $\times$ group.

\section{fMRI data: effect of selective attention on face-repetition effects}

The main effect of faces presented in task-relevant, versus task-irrelevant, locations (i.e., independent of repetition) identified bilateral mid-fusiform regions $(44,-50,-24$ and -42 , $-44,-28 ; \mathrm{Z} \geq 4.29 ; P<0.001$, uncorrected; Fig. $3 A$ ), whereas the opposite contrast (houses in task-relevant, vs. task-irrelevant, locations) identified bilateral parahippocampal cortex $(16,-52,6$ and $-24,-36,-14 ; \mathrm{Z} \geq 5.50 ; P<0.01$, corrected for whole brain). This replicates previous results using a similar task (Vuilleumier et al. 2001; Wojciulik et al. 1998) and demonstrates that subjects selectively processed the pair of stimuli at cued locations.

We next identified areas that showed fMRI face-repetition effects common to when faces occurred in attended or unattended locations, by performing a conjunction analysis across contrasts comparing first and second presentations under both attentional conditions in the placebo group. Areas showing repetition decreases under both levels of attention were bilateral inferior temporo-occipital cortex, left inferior prefrontal gyrus, and premotor areas (Table 1A, Fig. 3B). By contrast, superior temporal sulcus, middle occipital gyrus, and striatum showed greater repetition decreases for faces presented in attended versus unattended locations (Table $1 C$ ). We note that the inferior temporo-occipital regions showing a repetition effect independent of attention did not show a main effect of attention (Fig. 3B), and, conversely, the mid-fusiform peaks showing a main effect of attention did not show any repetition effects (Fig. 3A). Areas that showed repetition increases across both levels of attention included biparietal cortex and posterior cingulate (Table $1 B$ ), an effect also reported by Henson et al. (2002).

Finally, as our behavioral data suggested a performance advantage on house judgments when faces were repeated at task-irrelevant locations, we examined the contrast of first versus second presentations of task-irrelevant faces (i.e., when house judgments were performed), and restricted the analysis to house-selective regions (by masking with the main effect of house-attention, thresholded at $P<0.001$, uncorrected). This identified a left parahippocampal region $(-22,-32,-10 ; \mathrm{Z}=$ $4.09, P<0.05$, corrected) that showed increased activity with repetition of unattended face stimuli, presumably as the houses became more dominant when the same faces were ignored. This repetition enhancement was indeed specific to trials when house judgments were required (and faces ignored), as shown 


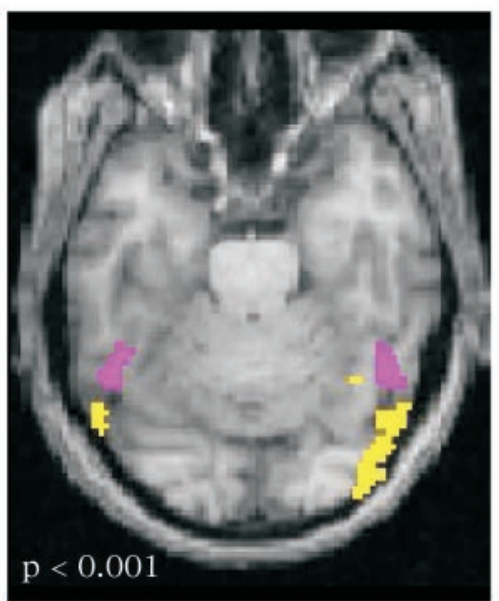

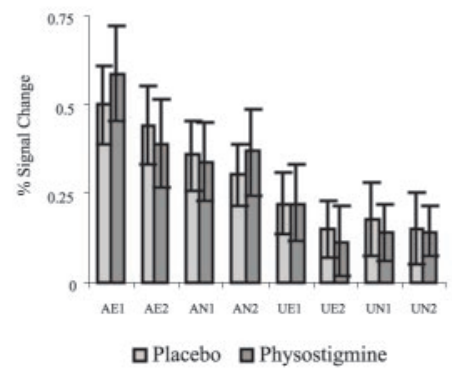

B R Inf. Occipital 40,-78,-18

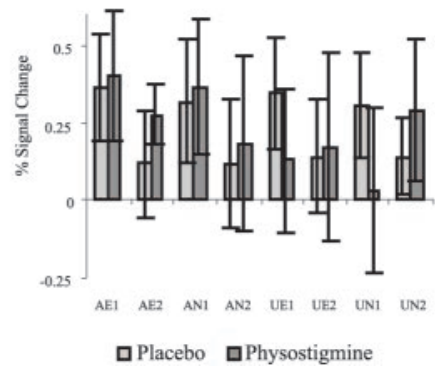

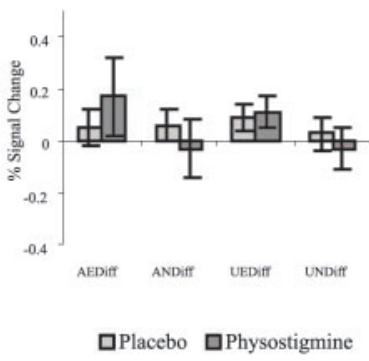

R Inf. Occipital 40,-78,-18

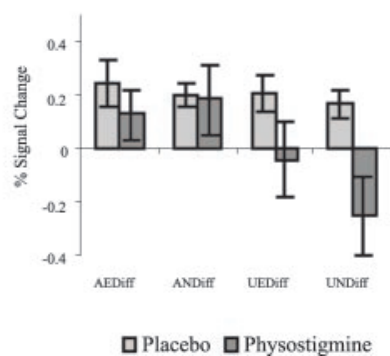

FIG. 3. Regions of inferior temporo-occipital cortex showing main effect of faces in attended vs. unattended locations, independent of repetition (mauve; $A$ ), and conjunction of repetition decreases to faces in both attended and unattended locations (yellow; $B$ ); both contrasts $P<0.001$, uncorrected. Activation map is superimposed on single-subject T1-weighted MRI brain, pitched to visualize inferior cortical surface. Graphs show percentage signal change from baseline in right mid-fusiform $(A)$ and inferior occipital cortex $(B)$ during first and second presentations of faces from each of 4 conditions (AE, AN, UE, UN). On right are plots of mean subject-specific signal differences between first and second presentations for each of 4 conditions (positive values represent repetition decreases; negative values represent repetition enhancements). Regions showing main effect of face-attention $(P<0.001)$ did not show a significant effect of repetition $(P>0.05)$, whereas regions showing repetitions to both attended and ignored faces $(P<0.001)$ did not show a significant effect of face-attention $(P>0.05)$. Placebo and physostigmine groups are shown separately. Left-sided plots are corrected for mean over all conditions between groups (no significant main effect of drug).

by a significant repetition $\times$ attention interaction $[F(1,14)=$ 5.0; $P<0.05]$.

\section{fMRI data: effect of emotion on face-repetition effects}

Comparison of signal estimates between emotional and neutral face conditions at the peak of the conjunction of facerepetition effects across attended and unattended contrasts suggested a similar repetition decrease in occipital cortex for both emotional and neutral faces (Fig. 3B). This was confirmed formally by performing the conjunction of priming effects between fearful and neutral-face trials, independent of attention (Table 2; yellow area in Fig. 4, graph A). The same posterior visual (and premotor) areas that showed face-repetition decreases for both levels of attention thus also showed repetition decreases to both neutral and emotional faces.

We next examined for repetition effects modulated by emotion (i.e., repetition $\times$ emotion interaction). This showed that left postero-inferior occipital cortex, as well as a region at posterior amygdala-hippocampal border, exhibited a greater repetition decrease for emotional, relative to neutral, stimuli (Table 2; blue area in Fig. 4, graph B). Conversely, the only area showing a greater repetition decrease to neutral, relative to emotional, faces was lateral orbitofrontal cortex (OFC: red area in Fig. 4). The pattern of repetition decreases in this region across conditions (Fig. 4A) parallels the RT priming effects observed behaviorally. Furthermore, a correlation analysis comparing the size of the emotional effect on RT priming with the size of the emotional effect on BOLD repetition decreases at the OFC peak $(38,38,-14, \mathrm{Z}=4.16)$ showed a positive trend $[r(28)=0.46, P=0.08]$, whereas this was significant $[r(28)=0.54, P=0.05]$ for the neighboring secondary peak $(40,46,-16 ; Z=4.14)$. Thus subjects showing the greatest attenuation of RT priming with emotional faces also tended to show the greatest diminution of repetition decreases in OFC toward emotional faces.

We note that none of the extrastriate areas exhibiting repetition effects showed a main effect of emotion, but such a main effect of emotion, independent of attention, was found in left fusiform ( $-40,-48,-24, \mathrm{Z}=3.27, P<0.001$, uncorrected), and medial amygdala-substantia innominata $(10,-8,-16$, $\mathrm{Z}=3.09, P=0.001$, uncorrected), in keeping with Vuilleumier et al. (2001).

\section{fMRI data: effects of cholinergic enhancement on repetition effects}

Given the role of cholinergic inputs on selective attention, we had hypothesized that physostigmine might enhance repetition-suppression effects (see Thiel et al. 2001, 2002a) specifically for attended faces (see also Furey et al. 2000a). Therefore we examined group $\times$ repetition interactions for attended and unattended trials separately. With faces at attended locations, this interaction identified left inferior occipital cortex as showing a greater repetition decrease under physostigmine relative to placebo $(-32,-66,-22 ; \mathrm{Z}=3.91 ; P<0.05$, 
TABLE 1. Common and differential effects of attention on priming in placebo group

\begin{tabular}{lrrrr}
\hline \hline \multicolumn{1}{c}{ Area } & & & & Z \\
& $\mathrm{X}$ & $\mathrm{y}$ & $\mathrm{z}$ & Value \\
\hline A. Repetition decreases to faces in both & attended & and unattended locations \\
Right Inferior Occipital & 40 & -78 & -18 & $6.25^{* *}$ \\
& 36 & -86 & -16 & $3.92^{*}$ \\
& 46 & -62 & -22 & $3.98^{*}$ \\
Right Inferior Temporal & 32 & -40 & -22 & 3.85 \\
Left Inferior Occipital & -48 & -62 & -22 & $4.49^{*}$ \\
Left Temporo-Parietal Junction & -54 & -40 & 34 & 4.09 \\
Right Lingual Gyrus & 12 & -56 & 6 & 3.94 \\
Cuneus & 6 & -86 & 14 & 4.34 \\
Left Inferior Frontal Gyrus & -52 & 38 & 8 & 4.21 \\
Right Inferior Frontal Gyrus & 60 & 24 & 10 & 3.29 \\
Left Posterior Superior Frontal Gyrus & -6 & 14 & 50 & 3.67 \\
Right Posterior Middle Frontal Gyrus & 58 & 16 & 30 & 3.95
\end{tabular}

B. Repetition increases to faces in both attended and unattended locations

$\begin{array}{lrrrr}\text { Right Parietal } & 54 & -38 & 56 & 4.52 \\ & 22 & -68 & 32 & 4.16 \\ \text { Left Parietal } & -32 & -56 & 30 & 3.42 \\ \text { Posterior Cingulate } & 8 & -34 & 18 & 3.67 \\ \text { Left Middle Temporal Gyrus } & -54 & 0 & -30 & 3.85 \\ \text { Right Premotor Cortex } & 14 & -8 & 68 & 4.05 \\ \text { Left Central Sulcus } & -24 & -16 & 44 & 3.70\end{array}$

C. Repetition decreases greater for faces in attended than unattended locations

$\begin{array}{lrrrr}\text { Left Superior Temporal Sulcus } & -62 & -36 & 12 & 4.26 \\ \text { Left Middle Occipital Gyrus } & -34 & -80 & 16 & 3.91 \\ \text { Right Striatum } & 22 & 8 & 0 & 3.68 \\ \text { Medial Orbitofrontal Cortex } \dagger & -2 & 42 & -22 & 4.20\end{array}$

Medial Orbitofrontal Cortex $\dagger$

D. Repetition decreases greater for faces in unattended than attended locations

No regions approached significance

$* P<0.05$ corrected for ROI (based on Henson et al., 2002; Jiang et al., 2000). ** $P<0.01$ corrected for whole brain. $†$ Regions also showing a three-way interaction of repetition $\times$ attention $\times$ emotion, that is, (AN1AN2)-(AE1-AE2)-[(UN1-UN2)-(UE1-UE2)].

corrected for ROI; Fig. 5). This occipital area also showed a repetition $\times$ attention $\times$ drug interaction $[F(1,28)=4.0 ; P<$ $0.05]$ that reflected physostigmine, but not placebo, engendering a greater repetition decrease for attended, versus ignored, faces [physostigmine: $F(1,28)=7.0 ; P<0.05$; placebo: $F(1,28)=0.6 ;$ ns]. Furthermore, in the same region, we note that physostigmine, versus placebo, resulted in reduced activity to the repeated face $[t(28)=2.4 ; P<0.05]$, but did not change activity to the first face $[t(28)=1.1 ; \mathrm{ns}]$. These results complement previous findings of modulation of repetition effects within left inferior occipital cortex by cholinergic blockade, when scopolamine resulted in a reduced repetition decrease, attributed specifically to an elevation in activity to the repeated stimulus (Thiel et al. 2001).

No regions showed attenuation of repetition decreases to faces at attended locations under physostigmine, relative to placebo, and no regions showed a significant drug $\times$ repetition interaction for faces at unattended locations.

We also tested for 3-way interactions of drug $\times$ repetition $\times$ condition (for conditions of attention and emotion, separately), given that these interactions showed significant effects in the RT data. We restricted our search volumes to those voxels showing a main effect of repetition decrease (regardless of attention and emotion condition) or a repetition $\times$ condition interaction in the placebo group (thresholded at $P<0.001$, uncorrected). Importantly, no areas showed a repetition $\times$ drug interaction over all conditions (i.e., there was no main effect of physostigmine on repetition independent of conditions).

In the repetition $\times$ drug $\times$ attention interaction, we found that the right inferior occipital region showing a maximum repetition decrease common to faces in both attended and unattended locations (as well as to both emotional and neutral faces) under placebo was found under physostigmine to show a repetition decrease selectively for attended faces only [i.e., repetition $\times$ attention $\times$ drug interaction: $F(1,28)=5.5 ; P<$ 0.05 ; a similar interaction was found in the left inferior occipital region showing a repetition $X$ drug interaction for attended faces; see above]. As shown in Fig. 3B, under physostigmine, the right inferior occipital region manifested a similar degree of repetition decrease for faces in attended locations as under placebo, but showed a trend for repetition increases for faces at unattended locations $[t(14)=1.7 ; P=0.1]$, in contrast to the placebo group. There were no regions in which this interaction survived correction for search volume.

Finally, the repetition $\times$ drug $\times$ emotion interaction revealed that the lateral orbitofrontal region $(38,38,-12)$, previously found in the placebo group to display less repetition effects with emotional, relative to neutral, faces, was not associated with this pattern under physostigmine $(\mathrm{Z}=4.51 ; P<$ 0.05 , corrected; Fig. $4 B$ ). In fact, physostigmine produced the opposite effect at this point, that is, greater repetition decreases with emotional than neutral faces $[F(1,28)=10.3 ; P<0.01]$.

TABLE 2. Common and differential effects of emotion on priming in placebo group

\begin{tabular}{|c|c|c|c|c|}
\hline Area & $\mathrm{x}$ & $\mathrm{y}$ & $\mathrm{z}$ & $\begin{array}{c}\mathrm{Z} \\
\text { Value }\end{array}$ \\
\hline \multicolumn{5}{|c|}{ A. Repetition decreases to both emotional and neutral faces } \\
\hline \multirow[t]{2}{*}{ Right Inferior Occipital } & 40 & -78 & -16 & $5.17 * *$ \\
\hline & 46 & -62 & -24 & $4.17 *$ \\
\hline Right Inferior Temporal & 32 & -52 & -22 & 3.42 \\
\hline Left Inferior Occipital & -46 & -62 & -22 & $4.57 *$ \\
\hline Left Middle Occipital Gyrus & -34 & -84 & 16 & 3.36 \\
\hline Left Temporo-Parietal Junction & -52 & -42 & 34 & 3.68 \\
\hline Cuneus & -2 & -86 & 6 & 4.02 \\
\hline Left Amygdala & -26 & 0 & -26 & 3.34 \\
\hline Right Posterior Middle Frontal Gyrus & 58 & 12 & 30 & 3.67 \\
\hline Left Posterior Superior Frontal Gyrus & -8 & 12 & 48 & 3.63 \\
\hline
\end{tabular}

B. Repetition increases to both emotional and neutral faces

Right Parietal

Posterior Cingulate

Left Hippocampus

Left Central Sulcus

Left Superior Frontal Sulcus

Right Middle Frontal Gyrus

$\begin{array}{rrrr}22 & -68 & 32 & 3.56 \\ 6 & -24 & 32 & 3.52 \\ -22 & -34 & -10 & 4.13 \\ -24 & -16 & 42 & 4.01 \\ -18 & 38 & 30 & 3.70 \\ 38 & 56 & -10 & 3.77\end{array}$

C. Repetition decreases greater for emotional than neutral faces

$\begin{array}{lllll}\text { Left Posterior Inferior Occipital } & -36 & -90 & -14 & 3.45\end{array}$ $\begin{array}{lllll}\text { Right Posterior Amygdala-Hippocampus } & 18 & -10 & -12 & 3.47\end{array}$

D. Repetition decreases greater for neutral than emotional faces

$\begin{array}{lllll}\text { Right Lateral Orbitofrontal Cortex } & 38 & 38 & -14 & 4.16 \\ & 40 & 46 & -16 & 4.14\end{array}$

$* P<0.05$ corrected for ROI (based on Henson et al., 2002; Jiang et al., 2000). *** $P<0.05$ corrected for whole brain. 


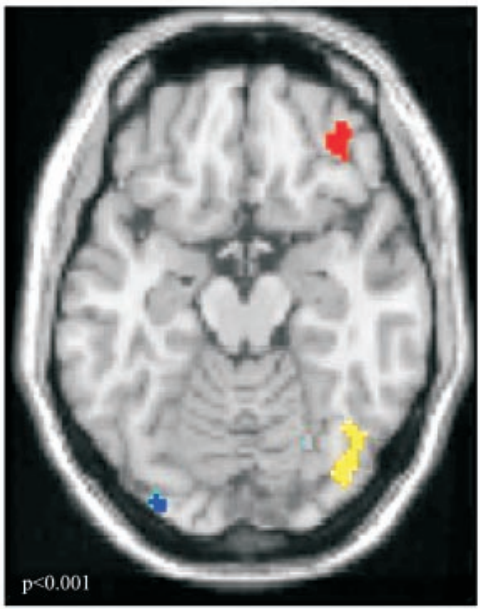

A

R OFC $38,38,-14$

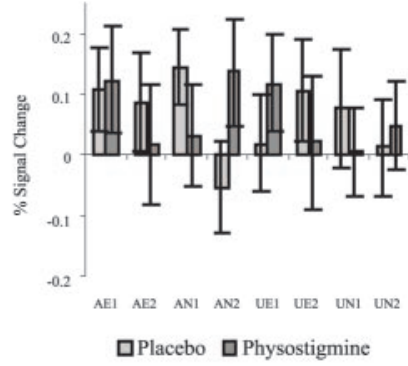

B L Post. Occipital $-36,-90,-14$

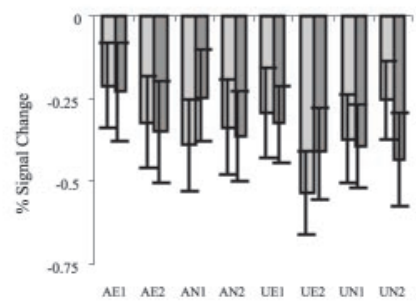

口Placebo 口Physostigmine
R OFC $38,38,-14$

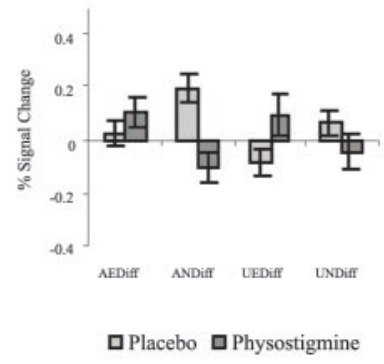

L Post. Occipital $-36,-90,-14$

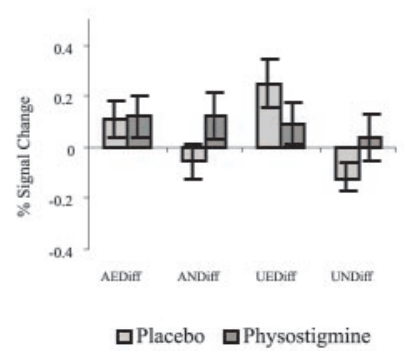

FIG. 4. Regions of inferior temporo-occipital and orbitofrontal cortex showing conjunction of repetition decreases for both emotional and neutral faces (yellow; activity plot similar to that shown in Fig. 3B; a left inferior occipital cluster occurred on lower slice); or greater repetition decreases to neutral, relative to emotional, faces (red; $A$ ); or greater repetition decreases to emotional, relative to neutral, faces (blue; $B$ ); all contrasts thresholded at $P<0.001$, uncorrected. Activation maps are superimposed on single-subject T1-weighted MRI brain $(\mathrm{z}=-14)$. Graphs show percentage signal change from baseline in right lateral orbitofrontal cortex (OFC: $A$ ), and left posterior occipital $(B)$ regions during first and second presentations of faces from each of 4 conditions (AE, AN, UE, UN). Scales differ between regions. On right are plots of mean subject-specific signal differences between first and second presentations for each of 4 conditions (positive values represent repetition decreases; negative values represent repetition enhancements). Placebo and physostigmine groups are shown separately for each contrast. Left-sided plots are corrected for mean over all conditions between groups (no significant main effect of drug).

Furthermore, the right OFC under physostigmine did not show any correlation between RT and BOLD measures of an emotion $\times$ repetition interaction $[r(28)=-0.12$ and $r(28)=$ -0.06 for the 2 peaks within OFC showing an emotion $\times$ repetition interaction under placebo], unlike the case under placebo (normalized differences from placebo correlation coefficients given by $\mathrm{z}=1.6$ and 1.7 , respectively, $P \leq 0.10$ for both).

\section{Eye tracking and adverse drug effects}

The frequency of saccades and median angular deviation of the eye were measured during 250-ms epochs before and after the onset of each stimulus. The mean percentage of trials with saccades over both epochs was only 3.2 and 3.0, under placebo and physostigmine, respectively, with no reliable group differences during the 2 peristimulus epochs $[F(1,22)=1.44$ and 1.78 , respectively; $P \geq 0.2$ ]. There were no significant effects interactions within a 4-way ANOVA with factors of group, repetition, attention, and emotion, during either of the 2 peristimulus periods, for either saccade number, or median ocular position (all values of $F<1.8 ; P \geq 0.2$ ).

Questionnaires detailing possible side effects and subjective feelings, as well as measures of blood pressure and pulse, were recorded before infusion and just before scanner entrance, when a steady state of physostigmine would be expected (Furey et al. 2000b). Although subjects given physostigmine with glycopyrrolate were more likely to experience a dry mouth $(\mathrm{U}=62, P<0.05)$ and dizziness $(\mathrm{U}=68, P<0.01)$, the mean intensity of these symptoms was very small $(1.3 \pm$ 0.95 and $0.5 \pm 0.40$, respectively, on a scale of 0 to 6$)$. Two subjects given physostigmine who vomited were excluded and replaced with alternative subjects. A pooled measure of subjective alertness (Bond and Lader 1974) suggested that the physostigmine group felt more drowsy at test relative to preinfusion [mean percentage difference between preinfusion and prescan $=-1.3 \pm 2.2 \%$ for placebo, and $+8.8 \pm 3.7 \%$ for physostigmine; $F(1,28)=6.4 ; P<0.05]$, although the simple effects of group for absolute subjective alertness at each time point were insignificant. There were no significant cardiovascular main effects or interactions.

\section{I S C U S S I O N}

\section{Effect of selective attention on repetition effects}

Previous functional imaging studies have shown decreased responses, predominantly in extrastriate cortical areas, with repetition of attended visual stimuli (e.g., Badgaiyan 2000; Buckner et al. 2000; Henson et al. 2002; Vuilleumier et al. 2002a). The present study demonstrates that repetition decreases in inferior temporo-occipital cortex can be independent of whether stimuli occur in attended (task-relevant) or ignored (task-irrelevant) locations. These results provide a neural counterpart to behavioral studies showing that perceptual priming can occur without the need for attention or awareness of stimuli at study phase (Parkin et al. 1990; Stone et al. 1998; Szymanski 


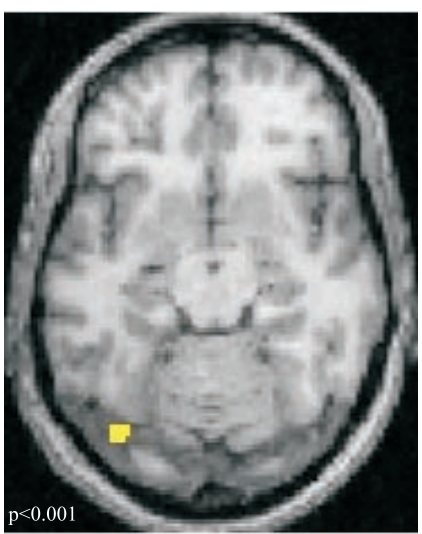

L Inf. Occipital -34,-68,-22

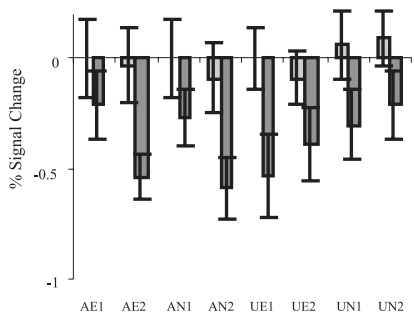

口Placebo 口Physostigmine
L Inf Occipital -34,-68,-22

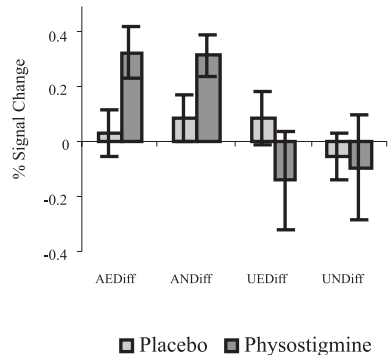

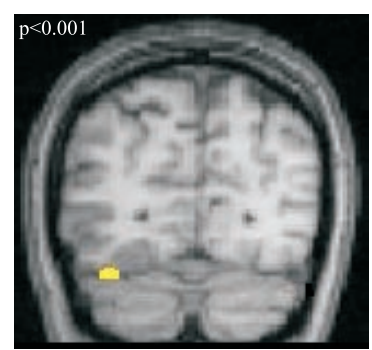

FIG. 5. Regions of inferior temporo-occipital cortex showing greater repetition decreases for physostigmine than placebo, specifically for faces in attended locations $(P<0.001$, uncorrected). Area showing this significant interation was close to region previously showing repetition decreases in face priming study (Henson et al. 2002), and to region previously showing diminution of repetition decrease under cholinergic blockade (Thiel et al. 2001). Activation map is superimposed on single-subject T1weighted MRI brain. Transverse slice taken at $\mathrm{z}=-22$; coronal slice taken at $\mathrm{y}=-68$. Graph shows percentage signal change from baseline in left inferior occipital cortex during first and second presentations of faces from each of 4 conditions (AE, AN, UE, $\mathrm{UN}$ ). On right are plots of mean subject-specific signal differences between first and second presentations for each of 4 conditions, at the same point. Placebo and physostigmine groups are shown separately for each contrast, and are not mean-corrected.

and MacLeod 1996; Vuilleumier et al. 2002b). Furthermore, our data support proposals that the neural mechanisms underlying repetition suppression reflect intrinsic properties of cell populations within temporo-occipital cortex that are expressed without top-down attentional facilitation (Desimone 1996; Wiggs and Martin 1998).

An earlier fMRI study using a working memory task similarly found that repetition decreases occurred in inferior temporal regions to both target and nontarget faces (Jiang et al. 2000). However, because both types of face were task-relevant, it is likely that attention to both types of stimuli would have initially been equal, and (may have) changed only after stimulus discrimination had been made. In the present task, attention was systematically manipulated by cueing subjects toward selective locations before presentation of brief multistimuli arrays. That this can be successfully achieved is suggested by a study with a similar procedure and stimuli (Vuilleumier et al. 2001) in which subjects were unaware of the identity and features of task-irrelevant, but not task-relevant, faces. Moreover, our own fMRI results show that attention-independent repetition effects and attention-dependent main effects in inferior temporo-occipital cortex may co-occur within the same task. The fact that these effects occurred, respectively, in posterior versus more anterior extrastriate regions (Fig. 3) is in line with the idea that "top-down" modulation of visual regions becomes increasingly influential at progressively later stages of stimulus processing (see Martinez et al. 2001).

Although these findings imply a degree of autonomy for repetition effects in sensory cortex, this need not imply that repetition effects in other areas cannot be modulated by atten- tional processes. Indeed, middle occipital and superior temporal cortex showed greater repetition decreases for faces when in task-relevant locations than in irrelevant locations. Similarly, the nature of the task can influence repetition effects in extrastriate cortex (Henson et al. 2002; Reber et al. 1998). Furthermore, electrophysiological studies have suggested that some repetition effects within inferior temporal regions do not occur within an "initial wave" of perceptual discrimination (i.e., $<200$ ms poststimulus; Nagy and Rugg 1989; Puce et al. 1999), but there remains uncertainty as to whether later repetition effects occur subsequent to (and possibly as a result of) activity within task-related frontal regions (Dale et al. 2001; Dhond et al. 2001). Although our study cannot resolve these issues of temporal order, we do show that repetition decreases may occur both independently and dependently upon "topdown" attentional factors in different brain areas, at least under conditions where repetition is incidental to task.

We note that repetition decreases were prominent in inferior occipital cortices but did not extend into the fusiform gyrus, in which there was a main effect of attention. This is consistent with a previous priming study that also used unfamiliar faces (Henson et al. 2002) and may reflect a functional separation of perception (which is repeated) versus recognition (which can occur only on the second presentation of a previously unfamiliar face) between occipital and fusiform regions, respectively (see Henson et al. 2000). Our results additionally show that attention-independent repetition effects may also occur in inferior frontal and premotor cortex, suggesting that automatic repetition effects can be expressed in higher processing centers, as well as in early visual regions. Note that repetition 
effects have previously been found to extend into frontoparietal regions even for unconscious, masked primes (Dehaene et al. 1998) again reflecting attention-independent processes (see Naccache and Dehaene 2001).

Our finding of similar fMRI repetition effects for faces presented in both task-relevant and task-irrelevant locations in inferior temporo-occipital cortex was mirrored behaviorally, in that the RT advantage observed for primed faces was not significantly different between the 2 attentional conditions. In the case of task-irrelevant face repetition, such speeding of responses occurred while subjects were judging (nonrepeated) house stimuli, and repetition effects could therefore reflect a habituation to specific distractors (e.g., Lorch et al. 1984). Thus repetition decreases may serve not only to facilitate processing of task-relevant stimuli (Wiggs and Martin 1998), but also to reduce interference from competing task-irrelevant stimuli (Desimone 1996; Kastner and Ungerleider 2001). The latter possibility is supported by our finding that the response in parahippocampal cortex to task-relevant houses was enhanced when particular faces were repeated at task-irrelevant locations.

\section{Effect of emotion on repetition effects}

We found that the RT advantage normally observed with repetition of neutral faces was significantly diminished for repetition of fearful faces, without a main effect of emotion on RT. A possible explanation is that the emotional properties of stimuli interfered with mechanisms involved in behavioral priming or habituation. Our imaging data enabled us to identify brain regions that exhibited an equivalent repetition $\times$ emotion interaction. Thus lateral orbitofrontal cortex (OFC) showed less repetition decrease specifically with fearful faces, mirroring the behavioral effect (Fig. 4A), as further evidenced by correlation analyses. Several findings accord with a role of this region in such emotion-sensitive reduction of priming. First, the OFC receives amygdala efferents (Amaral and Price 1984) and may relay the results of emotional evaluations within the amygdala to ongoing decision-making and motor-response centers within the prefrontal cortex (Bechara et al. 2000). Second, the pattern of activity in lateral OFC appears consistent with data implicating this region in response inhibition on the basis of emotional information (Elliot et al. 2000; Iversen and Mishkin 1970). Third, physostigmine was found to negate the effect of emotion on behavioral priming, while reversing the dependency of repetition decreases on emotion found within the same part of OFC (as discussed later).

In contrast to orbitofrontal cortex, extrastriate visual areas showed similar, or even greater, repetition decreases for emotional versus neutral faces (Fig. $4 B$ ). These regions were more posterior than the lateral fusiform sites showing increased responses to faces with attention or with fearful expression (see also Vuilleumier et al. 2001), again suggesting that repetition effects in occipital cortex occur largely independently of "topdown" attentional or emotional processing. However, the posterior occipital region showing greater repetition decreases for emotional faces might receive feedback influences from the amygdala, which activated as a main effect of emotion (see also Vuilleumier et al. 2001) and showed differential responses to first, versus repeated, presentation of fearful faces. In keeping with the latter finding, the amygdala also showed greater habituation to unpleasant than neutral faces in a recent fMRI experiment that controlled for physical differences of stimuli (Rotshtein et al. 2001).

\section{Effect of cholinergic enhancement on repetition effects}

Because cholinergic blockade with scopolamine has previously been found to inhibit both behavioral and neuronal correlates of repetition priming to attended stimuli (Thiel et al. 2001, 2002a), we expected to find an increase of both measures with the cholinergic enhancer physostigmine. Consistent with this prediction, physostigmine produced a greater repetition decrease in left inferior occipital cortex (selectively for faces in attended locations), although this did not significantly benefit RT or accuracy. This occipital area was close to those previously showing repetition decreases to faces (Henson et al. 2002; Jiang et al. 2000), or showing an elimination of repetition effects after cholinergic blockade (Thiel et al. 2001). The fact that no behavioral priming advantage was observed with physostigmine suggests that an additional rate-limiting step of the task may lie downstream from perceptual processes in occipital cortex (e.g., response-related).

The nature of the physostigmine interaction with repetition was closely complementary to that previously found with scopolamine (Thiel et al. 2001) in another respect: both cholinergic manipulations affected only response to the repeated occurrence of an item, not to the initial presentation. This contrasts with other drugs (e.g., GABAergic modulators) that disrupt cortical repetition effects and priming through effects on novel stimulus processing (Thiel et al. 2001; Vidailhet et al. 1999). Our results may also be relevant in the context of Alzheimer's disease (AD), in which perceptual priming is impaired (Schwartz et al. 1996; Shimamura et al. 1987). Because $\mathrm{AD}$ patients have shown paradoxical repetition enhancements in inferior occipital cortex, in contrast to healthy agematched subjects who show repetition decreases (Backman et al. 2000), we speculate that the beneficial effect of anticholinesterases on priming observed in AD (Riekkinen and Riekkinen 1999) may arise from an enhancement of repetition effects in inferior occipital cortex, as observed here in healthy subjects.

In contrast to the placebo group, who showed similar repetition decreases in inferior occipital regions for faces shown at either attended or ignored locations, we found in the physostigmine group that repetition decreases occurred preferentially to attended stimuli (Figs. 3B, 5B). These results complement our RT data in showing that physostigmine, but not placebo, induced a repetition benefit selectively with attended stimuli. Such an effect would be consistent with studies showing the importance of cortical cholinergic modulation on selective attention (Sarter et al. 2001), noise filtering (Sato et al. 1987), and selective perceptual processing of stimuli that need to be remembered (Furey et al. 2000).

Cholinergic modulation has also been shown to enhance processing of emotional stimuli (Holland and Gallagher 1999) and to facilitate experience-dependent cortical plasticity specifically to fear-conditioned stimuli (Ji et al. 2001; Thiel et al. 2002b; Weinberger et al. 1998). Our results extend the role of acetylcholine in emotional learning by showing that physostigmine favored repetition decreases to emotional, relative to neutral, faces in orbitofrontal cortex (thus reversing the normal 
pattern); negated the detrimental effect of emotion on primed RTs seen under placebo; and tended to eliminate any behavioral-BOLD correlation within OFC found under placebo. The OFC has especially strong interconnections with cholinergic neurons of the nucleus basalis (Cavada et al. 2000) and is modulated by acetylcholine during reinforcement learning in animals (Aou et al. 1983).

In conclusion, our experiment has shown that repetition priming, and its associated hemodynamic marker of extrastriate cortex repetition decrease, can occur by a similar amount for stimuli appearing at task-relevant or -irrelevant locations. In contrast, emotional stimuli reduced behavioral priming, associated with an attenuation of repetition decreases in lateral orbitofrontal cortex. Additionally, we demonstrated that cholinergic enhancement can augment the size of repetition effects in inferior occipital cortex, and favor behavioral and neural priming effects for both attended and emotional stimuli (the latter being attributed to a reversal of the emotion-selective impairment of priming in untreated subjects). These results suggest that cortical mechanisms underlying priming may occur automatically (in extrastriate areas) and yet be influenced by intrinsic stimulus value (in orbitofrontal cortex) and cholinergic modulation (in both regions).

We thank J. Winston and R. Henson for useful comments. The Karolinska Directed Emotional Faces pictoral image set that we used for this study is available from the Dept. of Clinical Neurosciences, Karolinska Hospital, Stockholm, Sweden (http://www.ki.se/cns).

\section{I S C L O S URE S}

This work was supported by program grants from the Wellcome Trust to R. J. Dolan and J. Driver, and a research grant from the German Research Foundation (Deutsche Forschungsgemeinschaft) to C. M. Thiel.

\section{REFERENCES}

Amaral DG and Price JL. Amygdalo-cortical projections in the monkey (Macaca fascicularis). J Comp Neurol 230: 465-496, 1984.

Aou S, Oomura Y, and Nishino H. Influence of acetylcholine on neuronal activity in monkey orbitofrontal cortex during bar press feeding task. Brain Res 275: 178-182, 1983.

Backman L, Almkvist O, Nyberg L, and Andersson J. Functional changes in brain activity during priming in Alzheimer's disease. J Cogn Neurosci 12: 134-141, 2000.

Badgaiyan RD. Neuroanatomical organization of perceptual memory: an fMRI study of picture priming. Hum Brain Mapp 10: 197-203, 2000.

Badgaiyan RD, Schacter DL, and Alpert NM. Priming within and across modalities: exploring the nature of $\mathrm{rCBF}$ increases and decreases. Neuroimage 13: 272-282, 2001.

Bechara A, Damasio H, and Damasio AR. Emotion, decision making and the orbitofrontal cortex. Cereb Cortex 10: 295-307, 2000.

Bentley P, Vuilleumier P, Thiel CM, Driver J, and Dolan RJ. Cholinergic enhancement modulates neural correlates of selective attention and emotional processing. Neuroimage In press.

Bond $\mathbf{A}$ and Lader $\mathbf{M}$. The use of analogue scales in rating subjective feelings. Br J Med Psychol 47: 211-218, 1974.

Brown MW and Xiang JZ. Recognition memory: neuronal substrates of the judgement of prior occurrence. Prog Neurobiol 55: 149-189, 1998.

Buckner RL, Goodman J, Burock M, Rotte M, Koutstaal W, Schacter D, Rosen B, and Dale AM. Functional-anatomic correlates of object priming in humans revealed by rapid presentation event-related fMRI. Neuron 20: 285-296, 1998.

Cavada C, Company T, Tejedor J, Cruz-Rizzolo RJ, and Reinoso-Suarez F. The anatomical connections of the macaque monkey orbitofrontal cortex. A review. Cereb Cortex 10: 220-242, 2000.
Dale AM, Liu AK, Fischl BR, Buckner RL, Belliveau JW, Lewine JD, and Halgren E. Dynamic statistical parametric mapping: combining fMRI and MEG for high-resolution imaging of cortical activity. Neuron 26: 55-67, 2000.

Dehaene S, Naccache L, Le Clec'H G, Koechlin E, Mueller M, DehaeneLambertz G, van de Moortele P, and Le Bihan D. Imaging unconscious semantic priming. Nature 395: 597-600, 1998.

Desimone R. Neural mechanisms for visual memory and their role in attention. Proc Natl Acad Sci USA 93: 13494-13499, 1996.

Dhond RP, Buckner RL, Dale AM, Marinkovic K, and Halgren E. Spatiotemporal maps of brain activity underlying word generation and their modification during repetition priming. J Neurosci 21: 3564-3571, 2001.

Elliott R, Dolan RJ, and Frith CD. Dissociable functions in the medial and lateral orbitofrontal cortex: evidence from human neuroimaging studies. Cereb Cortex 10: 308-317, 2000

Friston KJ, Fletcher P, Josephs O, Holmes A, Rugg MD, and Turner R. Event-related fMRI: characterizing differential responses. Neuroimage 7: 30-40, 1998.

Friston KJ, Holmes AP, Worsley KJ, Poline JB, Frith CD, and Frackowiak RSJ. Statistical parametric maps in functional imaging: a general linear approach. Hum Brain Mapp 2: 189-210, 1995.

Furey ML, Pietrini P, Alexander GE, Mentis MJ, Szczepanik J, Shetty U, Greig NH, Holloway HW, Schapiro MB, and Freo U. Time course of pharmacodynamic and pharmacokinetic effects of physostigmine assessed by functional brain imaging in humans. Pharmacol Biochem Behav 66: 475-481, 2000b.

Furey ML, Pietrini P, and Haxby JV. Cholinergic enhancement and increased selectivity of perceptual processing during working memory. Science 290: 2315-2319, 2000a.

Furey ML, Pietrini P, Haxby JV, Alexander GE, Lee HC, VanMeter J, Grady CL, Shetty U, Rapoport SI, Schapiro MB, and Freo U. Cholinergic stimulation alters performance and task-specific regional cerebral blood flow during working memory. Proc Natl Acad Sci USA 94: 65126516, 1997.

Glaser D, Penny W, Henson R, and Friston K. Coping with the variance structure of neuroimaging data (Abstract). Proc Cogn Neurosci Soc 141: E92, 2002.

Henson R, Shallice T, and Dolan R. Neuroimaging evidence for dissociable forms of repetition priming. Science 287: 1269-1272, 2000.

Henson R, Shallice T, Gorno-Tempini ML, and Dolan R. Face repetition effects in implicit and explicit memory tests as measured by fMRI. Cereb Cortex 12: 178-186, 2002.

Holland PC and Gallagher M. Amygdala circuitry in attentional and representational processes. Trends Cogn Sci 3: 65-73, 1999.

Iversen SD and Mishkin M. Perseverative interference in monkeys following selective lesions of the inferior prefrontal convexity. Exp Brain Res 11: 376-386, 1970.

Ji W, Gao E, and Suga N. Effects of acetylcholine and atropine on plasticity of central auditory neurons caused by conditioning in bats. J Neurophysiol 86: 211-225, 2001.

Jiang Y, Haxby JV, Martin A, Ungerleider LG, and Parasuraman R. Complementary neural mechanisms for tracking items in human working memory. Science 287: 643-646, 2000.

Kastner S and Ungerleider LG. The neural basis of biased competition in human visual cortex. Neuropsychologia 39: 1263-1276, 2001.

LaBar KS and Phelps EA. Perceptual priming of emotionally-arousing scenes is spared following bilateral amygdala damage (Abstract). In: The Amygdala in Brain Function: Basic and Clinical Approaches, Galveston, TX, 2002.

Lorch EP, Anderson DR, and Well AD. Effects of irrelevant information on speeded classification tasks: interference is reduced by habituation. $J$ Exp Psychol Hum Percept Perform 10: 850-864, 1984.

Lundh LG and Ost LG. Explicit and implicit memory bias in social phobia. The role of subdiagnostic type. Behav Res Ther 35: 305-317, 1997.

Lundqvist D, Flykt A, and Vhman A. The Karolinska Directed Emotional Faces, 1998. [Pictoral face set available from Department of Neurosciences, Karolinska Hospital, Stockholm, Sweden.].

Martinez A, DiRusso F, Anllo-Vento L, Sereno MI, Buxton RB, and Hillyard SA. Putting spatial attention on the map: timing and localization of stimulus selection processes in striate and extrastriate visual areas. Vision Res 41: 1437-1457, 2001.

Morris JS, Buchel C, and Dolan RJ. Parallel neural responses in amygdala subregions and sensory cortex during implicit fear conditioning. Neuroimage 13: 1044-1052, 2001. 
Naccache $\mathbf{L}$ and Dehaene $\mathbf{S}$. The priming method: imaging unconscious repetition priming reveals an abstract representation of number in the parietal lobes. Cereb Cortex 11: 966-974, 2001.

Nagy ME and Rugg MD. Modulation of event-related potentials by word repetition: the effects of inter-item lag. Psychophysiology 26: 431-436, 1989.

Parkin AJ, Reid TK, and Russo R. On the differential nature of implicit and explicit memory. Mem Cognit 18: 507-514, 1990.

Price CJ and Friston KJ. Cognitive conjunction: a new approach to brain activation experiments. Neuroimage 5: 261-270, 1997.

Puce A, Allison T, and McCarthy G. Electrophysiological studies of human face perception. III. Effects of top-down processing on face-specific potentials. Cereb Cortex 9: 445-458, 1999.

Rasmusson DD. The role of acetylcholine in cortical synaptic plasticity. Behav Brain Res 115: 205-218, 2000.

Reber PJ, Stark CE, and Squire LR. Contrasting cortical activity associated with category memory and recognition memory. Learn Mem 5: 420-428, 1998.

Riekkinen P Jr. and Riekkinen M. THA improves word priming and clonidine enhances fluency and working memory in Alzheimer's disease. Neuropsychopharmacology 20: 357-364, 1999.

Rorden C and Brett M. Stereotaxic display of brain lesions. Behav Neurol 12: 191-200, 2000.

Rotshtein P, Malach R, Hadar U, Graif M, and Hendler T. Feeling or features: different sensitivity to emotion in high-order visual cortex and amygdala. Neuron 32: 747-757, 2001.

Sarter M, Givens B, and Bruno JP. The cognitive neuroscience of sustained attention: where top-down meets bottom-up. Brain Res Brain Res Rev 35 146-160, 2001.

Sato H, Hata Y, Masui H, and Tsumoto T. A functional role of cholinergic innervation to neurons in the cat visual cortex. J Neurophysiol 58: 765-780, 1987.

Schacter DL and Buckner RL. Priming and the brain. Neuron 20: 185-195, 1998.

Schwartz BL, Hashtroudi S, Herting RL, Schwartz P, and Deutsch SI. d-Cycloserine enhances implicit memory in Alzheimer patients. Neurology 46: 420-424, 1996

Shimamura AP, Salmon DP, Squire LR, and Butters N. Memory dysfunction and word priming in dementia and amnesia. Behav Neurosci 101: 347-351, 1987.

Stone M, Ladd SL, Vaidya CJ, and Gabrieli JD. Word-identification priming for ignored and attended words. Conscious Cogn 7: 238-258, 1998.
Szymanski KF and MacLeod CM. Manipulation of attention at study affects an explicit but not an implicit test of memory. Conscious Cogn 5: 165-175, 1996

Thiel CM, Friston KJ, and Dolan RJ. Cholinergic modulation of experiencedependent plasticity in human auditory cortex. Neuron 35: 567-574, 2002 b.

Thiel CM, Henson RN, and Dolan RJ. Scopolamine but not lorazepam modulates face repetition priming: a psychopharmacological fMRI study. Neuropsychopharmacology 27: 282-292, 2002a.

Thiel CM, Henson RN, Morris JS, Friston KJ, and Dolan RJ. Pharmacological modulation of behavioral and neuronal correlates of repetition priming. J Neurosci 21: 6846-6852, 2001.

Thiel CM, Muller CP, Huston JP, and Schwarting RKW. High versus low reactivity to a novel environment: behavioural, pharmacological and neurochemical assessments. Neuroscience 93: 243-251, 1999.

van Turennout M, Ellmore T, and Martin A. Long-lasting cortical plasticity in the object naming system. Nat Neurosci 3: 1329-1334, 2000.

Vidailhet P, Danion JM, Chemin C, and Kazes M. Lorazepam impairs both visual and auditory perceptual priming. Psychopharmacology (Berl) 147: 266-273, 1999.

Vuilleumier P, Armony JL, Driver J, and Dolan RJ. Effects of attention and emotion on face processing in the human brain: an event-related fMRI study. Neuron 30: 829-841, 2001.

Vuilleumier P, Henson RN, Driver J, and Dolan RJ. Multiple levels of visual object constancy revealed by event-related fMRI of repetition priming. Nat Neurosci 5: 491-499, 2002a.

Vuilleumier P, Schwartz S, Clarke K, Husain M, and Driver J. Testing memory for unseen visual stimuli in patients with extinction and spatial neglect. J Cogn Neurosci 14: 875-886, 2002 b.

Watkins PC, Martin CK, and Stern LD. Unconscious memory bias in depression: perceptual and conceptual processes. J Abnorm Psychol 109: 282-289, 2000.

Weinberger NM. Physiological memory in primary auditory cortex: characteristics and mechanisms. Neurobiol Learn Mem 70: 226-251, 1998.

Wiggs CL and Martin A. Properties and mechanisms of perceptual priming. Curr Opin Neurobiol 8: 227-233, 1998.

Wojciulik E, Kanwisher N, and Driver J. Covert visual attention modulates face-specific activity in the human fusiform gyrus: fMRI study. J Neurophysiol 79: 1574-1578, 1998.

Worsley KJ, Marrett S, Neelin P, Vandal AC, Friston KJ, and Evans AC. A unified statistical approach to determining significant signals in images of cerebral activation. Hum Brain Mapp 4: 58-73, 1996. 